\title{
Industrial Production of RHIC Magnets
}

\author{
M. D. Anerella, D. H. Fisher \\ Brookhaven National Laboratory, Upton, New York 11973, USA \\ E. Sheedy, T. McGuire \\ Northrop Grumman Corporation, Bethpage, New York 1.1714, USA
}

\begin{abstract}
RHIC $8 \mathrm{~cm}$ aperture dipole magnets and quadrupole cold masses are icing built for Brookhaven National Laboratory (BNL) by Northrop-Grumman Corporation at a production rate of one dipole magnet and two quadrupole cold masses per day. This work was preceded by a lengthy Technology Transfer effort which is described elsewhere [1]. This paper describes the tooling which is being used for the construction effort, the production operations at each workstation, and also the use of trend plots of critical construction parameters as a tool for monitoring performance in production. A report on the improvements to production labor since the start of the programs is also provided. The magnet and cold mass designs, and magnetic test results are described in more detail in a separate paper [2].
\end{abstract}

\section{INTRODUCTION}

Northrop Grumman Corporation (NGC) is presently working under two separate contracts to build a total of 373 $8 \mathrm{~cm}$ dipole magnets and $4328 \mathrm{~cm}$ quadrupole cold masses for the Relativistic Heavy Ion Collider (RHIC) at BNL. Crosssections of the dipole cold mass and completed magnet are shown in Figs. 1 and 2, respectively. (Quadrupole cold mass design is similar to the dipole.) The contracts were awarded on a competitive basis, considering both cost and technical merit of the proposals submitted by various companies, based on the

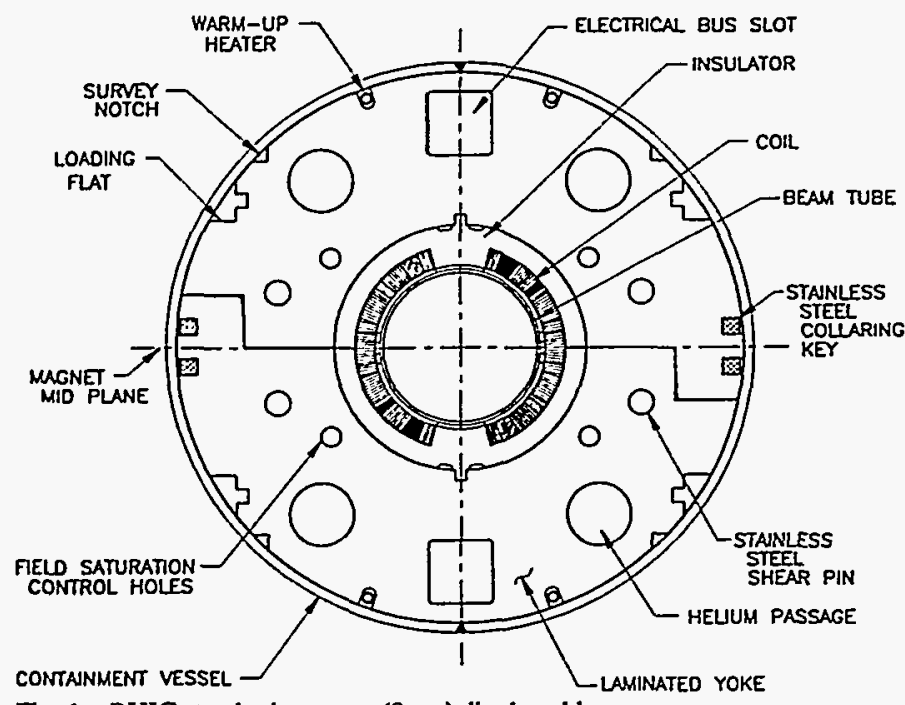

Fig. 1. RHIC standard aperture $(8 \mathrm{~cm})$ dipole cold mass.

Manuscript received June 12, 1995.

This work supported in part by the U.S. Department of Energy under Contract No. DE-AC02-76CH0OO16.

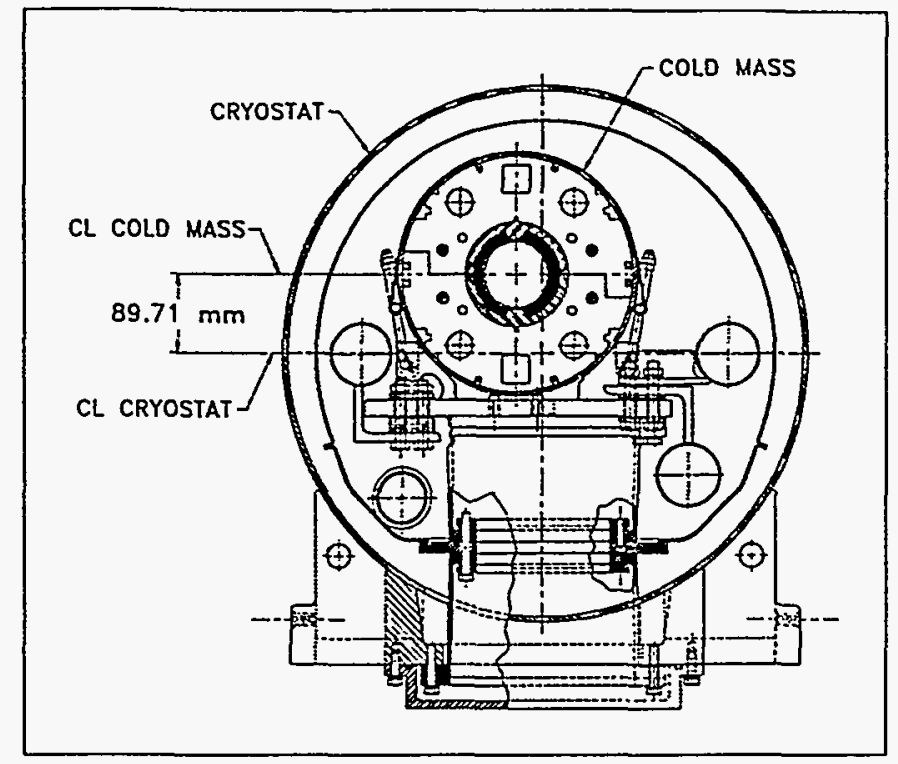

Fig. 2. A cross section of the RHIC arc dipole showing the asymmetrically located cold mass in the cryostat.

drawings and specifications supplied by BNL in the Request for Proposals. Both contracts are made up of three phases; Phase 1 , where tooling design and development, and industrial development of arc magnets/cold masses are completed; Phase 2 , where the balance of arc magnets/cold masses are built, and Phase 3, where the special length elements for the accelerator insertion regions are built. The dipole contract structure is "Cost Plus Fixed Fee" (CPFF) in Phase 1 and "Fixed Price Incentive Fee" (FPIF) in Phases 2 and 3. The quadrupole contract is FPIF in all three phases. For reasons of economy, no prototype or preproduction magnets have been made by NGC.

\section{TOOLING AND PRODUCTION DESCRIPTION}

Superconducting cable, which is supplied by BNL, is insulated at NGC using an insulating line constructed and supplied by BNL. The insulating line wraps the cable with two separate wraps of polyimide adhesive coated Kapton [3] with $50 \%$ overlap each. The line insulates cable at a maximum rate of twenty feet per minute, while continuously checking for Iumps and electrical shorts. A separate fixture designed and built by NGC wraps copper wedges one at a time with insulation identical to the cable insulation. A third workstation, also designed and built by NGC, satisfies all of the Kapton wrapping requirements for beam tubes, bus bars, and warm up heaters, operating at a rate of 16 feet per minute. For beam 
tubes, the fixtures also heat seal the Kapton to itself around the tubes. For warmup heaters, the wrapper also applies a final wrap of copper foil around the heaters. All NGC tool designs were reviewed and approved by BNL prior to fabrication.

Insulated cable and wedges, along with coil end parts, are wound into dipole coils using a winding machine purchased and inodified from the Noell Corporation. The winding machine was previously used to wind dipole coils for HERA. To adapt the machine to RHIC dipole coils, it was necessary to extend the mandrel supports slightly and space the cable carriage away from the machine to allow clearance between it and the mandrel supports at the coil ends. Reprogramming was also necessary to utilize the feature of automatic winding. For quadrupole coils, a winding machine provided by BNL is utilized. Both machines are of the "race-track" type, with carriage rotating about the stationary fixture, and mandrel rotating in conjunction with the carriage travel.

After coils are wound, they are relocated to a tool which rotates the mandrel so that a spiral wrap of high temperature Teflon [3] may be applied. This material is heat-shrinkable so as to hold the coil firmly in place until it is installed in the formblock. It also serves as a mold release after the coil is molded. Both dipole and quadrupole tools were designed and built by NGC.

Coil are then installed into the precision formblocks to be molded. The formblocks are made from prehardened tool steel (Viscount 44) and are assembled with seals that can withstand the $225^{\circ} \mathrm{C}$ cycle required to fuse the polyimide insulation to itself. Formblocks for both programs were designed by NGC and fabricated by a subcontractor. Included in the designs are easy conversions to the different Phase 3 coil lengths.

After installation into the formblock, coils are rolled into a precision hydraulic press for the molding operation. The presses are of the "window frame" design, with large cylinders straddling frames on roughly 1 meter spacing, and thick platens running continuously for the full press length. For the dipole program, the same press serves to both mold coils and collar yoke assemblies; conveyors feed the press from both ends to accomplish this. For the quadrupole program due to rate requirements, two coils are installed end to end and molded simultaneously in the press. (This solution is convenient since in the case of the quadrupole program, some of the Phase 3 coils are longer than the arc quadrupoles, and therefore require a longer press anyway.) A similar press is used exclusively for quadrupole yoke collaring. All presses were designed and fabricated by the same subcontractor, to a specification devised by NGC and approved by BNL. During molding, pressure, time and temperature are all monitored continuously by computer to assure that requirements are being met.

After curing, coils are inspected for azimuthal size, length, and electrical integrity. The same fixture, with different pole inserts, measures azimuthal size for both dipole and quadrupole coils. The two sides of each coil are measured simultaneously, with independent LVDTs recording the measurement for each side. The inspections are made efficient by the use of automated test carts, which run tests and log all mechanical and electrical data directly into the computer system. The fixture and test carts were designed and built by NGC.

After inspection, coils are readied for collaring by soldering copper stabilizers to the coil leads and adding preformed Kapton caps to the coil midplanes. To preserve mechanical integrity and therefore proper prestress at the leads, soldering is done to an accuracy of $0.125 \mathrm{~mm}$. For handling ease, preformed Kapton midplane caps have a dry, "b-staged" adhesive on their inner surface, and are secured to the coil by the use of a hand iron.

Coils are then installed into the iron yoke collars along with RX630 insulators, pole shims, coil shim supports, and an insulated beam tube, as follows. The lower yoke half (preweighed and sorted) is stacked onto the collaring contact tooling. The lower coil insulators are then installed into the yoke. Next, the lower coil (or coils for quadrupole) is (are) installed, followed by the pole shims, shim supports, and beam tube. (Beam tubes are installed for the dipole only; note that for quadrupole cold masses the beam tube is installed at BNL, and so in its place at NGC the coils are assembled around an inflatable, pressurized mandrel, supplied by BNL.) Next, the upper coil(s), which has (have) been pre-assembled to the shims and insulators, is (are) installed onto the lower coil(s). Finally, the upper yoke is stacked over the lower yoke and the cold mass is installed into the press. During collaring, applied load versus yoke closure is monitored and plotted, as an empirical check on the final prestress in the coils.

After collaring, quadrupole cold masses undergo end plate assembly, where coil end force is applied, and then final electrical assembly and test. Following the final electrical test, quadrupoles are shipped to BNL in NGC designed and built shipping containers.

Dipole collared yoke assemblies are removed from the press, rotated $90^{\circ}$ and then installed in the shell welding press. The stainless steel containment shells are then welded around the yoke as follows: First, one shell half is placed over the yoke assembly. Pneumatic cylinders push against the shell through roller bearing cradle assemblies and conform the yoke to continuous parallel rails of the nominal sagitta $(48.5 \mathrm{~mm}$ over $9.7 \mathrm{~m}$ ). The shell half is then continuously fillet welded directly to the iron yoke on both sides. The cold mass is then lifted, and intermittent "sagitta" supports are engaged. These supports contact the yoke survey notches through access holes in the half shells. The second shell half is placed over the sagitta supports and the cold mass is returned to the fixture. The pneumatic cylinders are re-engaged from above and below, conforming the cold mass to the sagitta supports, and the unwelded shell half to the yoke. The two shell halves are then welded together at the mating seam on each side. All welding is done by a single operator by the automatic Tig welding process. The automatic welding equipment was designed and fabricated by a subcontractor to a specification devised by NGC and approved by BNL. The press itself was designed and built by NGC. 


\section{DISCLAIMER}

Portions of this document may be illegible in electronic image products. Images are produced from the best available original document. 
After shell welding, the dipole cold mass is moved to an inspection station where sagitta and twist are checked using the shell access holes to measure directly to survey notches in the yoke. At the same station, the cold mass cradles are installed and welded to precise agreement with the average field angle of the cold mass as determined by the yoke twist measurements. At the same station, end plates are also located and tack welded in place. All equipment at this workstation was designed and built by NGC.

The cold mass is then moved to the next workstation where the end plate welding is completed. This welding is done using an automatic orbital Tig welder, fabricated by the same company which provided the shell welding equipment. Afterwards, at the same station, subassembled bus and warmup heaters are installed. Coil end force is applied and then final electrical installation is completed. Finally, shell access holes are covered by either welded patches or welded fiducial sockets, and end volume assemblies are welded on to complete the helium vessel. All of the equipment at this workstation was designed and built by NGC with the exception of the orbiter welder as mentioned, and also some of the electrical soldering equipment, which was developed and provided by BNL.

After the welding is completed, the cold mass is pressure tested to $345 \mathrm{psig}(23 \mathrm{~atm})$ and vacuum leak checked to $2 \mathrm{x}$ $10^{-10} \mathrm{std} \mathrm{cc} \mathrm{He} / \mathrm{sec}$. The helium mass spectrometer used was purchased from a subcontractor; all other associated equipment was designed and built by NGC. Of particular interest are the test fittings; since only $20 \%$ of the dipoles are planned to be cold tested at BNL, $80 \%$ of the magnets do not have test flanges. Therefore pressure and leak testing must be accomplished by sealing in these instances to the outside diameter of the end volume assembly tubes.

Following leak check, the cold mass is then pre-assembled to the upper support posts and heat shield. Multi-layer insulating blankets are installed and the cold mass is pulled into the vacuum vessel by chain and winch on a tow plate. Once installed, the cold mass is lifted by hydraulic jacks, the tow plate is removed, and the installation to the vacuum vessel is completed by bolting upper support posts to lower support posts and lower support posts to vacuum vessel. All equipment in this workstation was designed and built by NGC.

The magnet is then moved to the final inspection station, where survey measurements of cold mass and vacuum vessel fiducials are made using an optical theodolite system (existing equipment at NGC.) At the same station, warm magnetic measurements are completed using a rotating coil ("MOLE") system provided by BNL. The result from these magnetic measurements are forwarded electronically and reviewed by BNL before magnet shipment is approved. When completed, the magnet is installed onto shock mounts on an air-ride trailer for shipment to BNL. Restraints are installed into the vacuum vessel ports to restrain the cold mass, and protect the plastic support posts from shipping loads. The shock mount system and the shipping restraints were designed and fabricated by NGC.

\section{PRODUCTION IMPROVEMENTS}

The philosophy (and budget) of the NGC production floor requires that with each successive magnet built, less labor is required, according to a prescribed formula referred to as a learning curve. This reduction in labor is achieved partly by an operator becoming more proficient at a repetitive task. However, a significant part of the reduction comes from improvements to the process which make production more efficient. It is assumed at the outset that with exprrience, the operators will recognize these possible improvements. To ensure that all possible advantages are realized, NGC has adopted a formal approach to reviewing and implementing changes. Called Producibility Reviews, they are regular meetings where suggestions are reviewed by a group for potential benefit and possible impact. Those accepted are presented to BNL for technical approval as necessary (such as deleting redundant electrical tests). Other improvements involve streamlining operations such as material routings and computer systems procedures, and do not involve any technical issues. The striking results of the learning and improvements during production may be seen in Fig. 3, where total labor hours versus dipole magnet sequence number are plotted.

\section{PRODUCTION DATA TRENDS}

Figs. 4 and 5 show coil azimuthal stress during molding for dipole and quadrupole coils, respectively. For dipole coils, after an initial settling-in period, data appears to be uneventful, except for some high values of stress which have been traced to sporadic instrumentation problems. The quadrupole coil stress data also appears uneventful. The molding cycle used is described in detail in another paper [4].

Figs. 6 and 7, respectively, represent coil azimuthal size measurements for dipole and quadrupole coils.

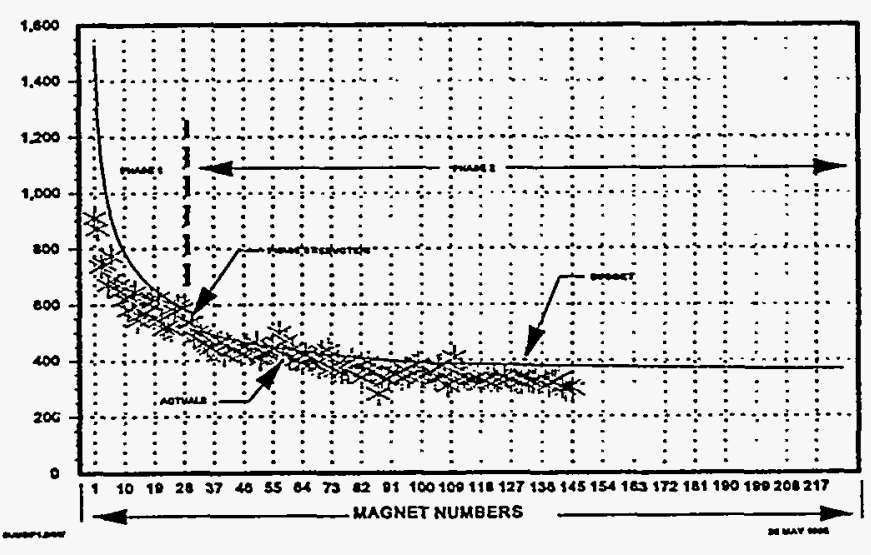

Fig. 3. RHIC dipole magnet program. Unit performance - production labor hours. All work stations - lot fabrication \& assembly. 


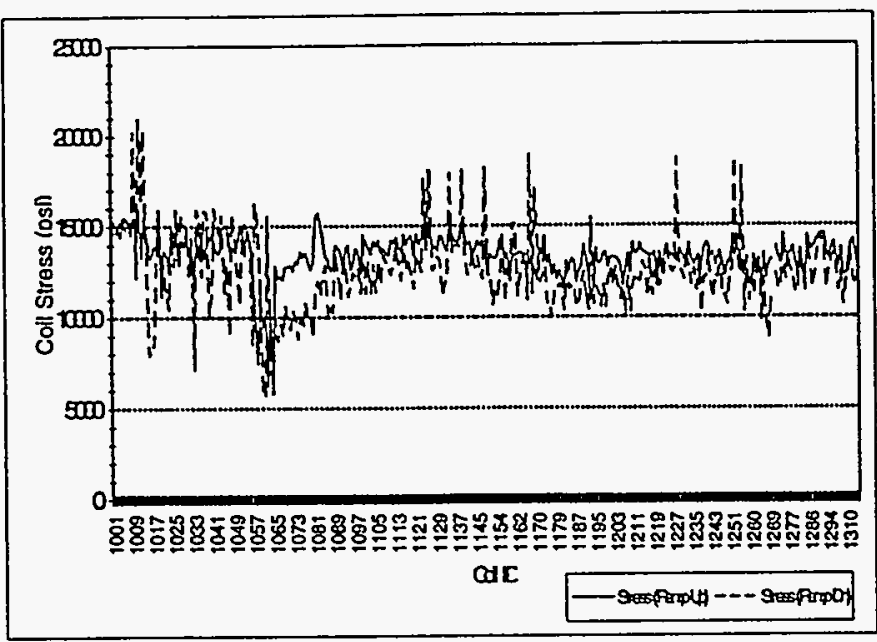

Fig. 4. RHIC $8 \mathrm{~cm}$ Dipole Curing Azimuthal Stress Data.

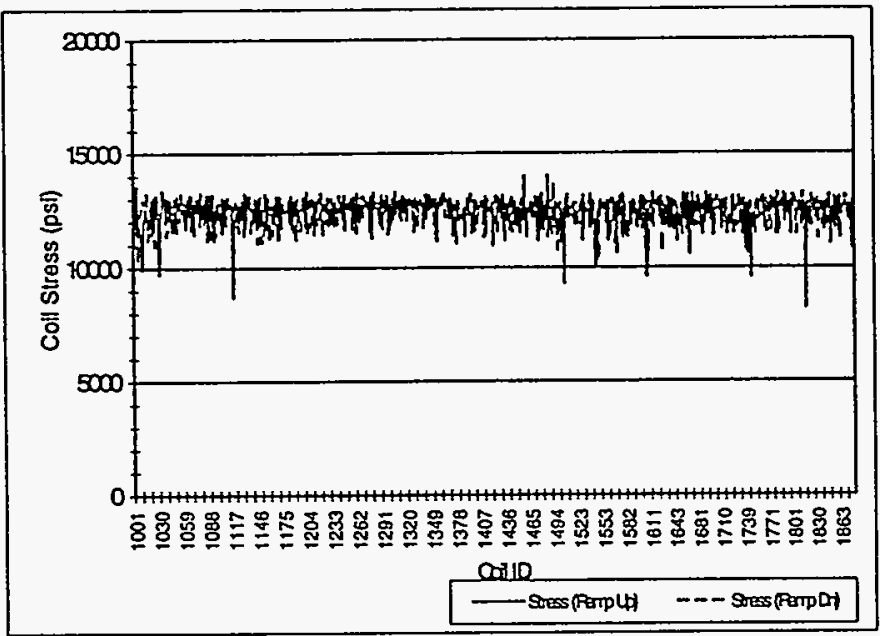

Fig. 5. RHIC $8 \mathrm{~cm}$ Quadrupole Curing Azimuthal Stress Data.

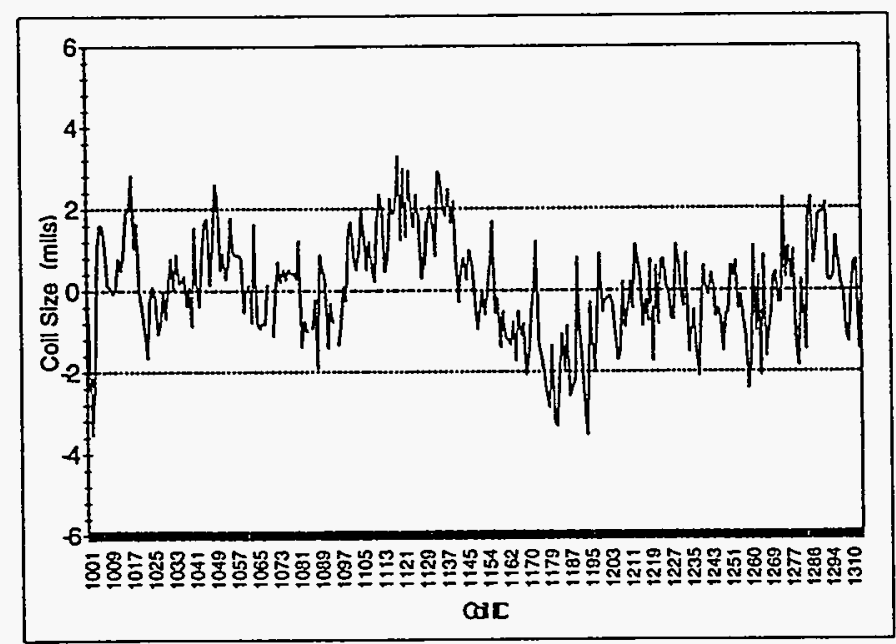

Fig. 6. RHIC $8 \mathrm{~cm}$ Dipole Mean Coil Azimuthal Size.

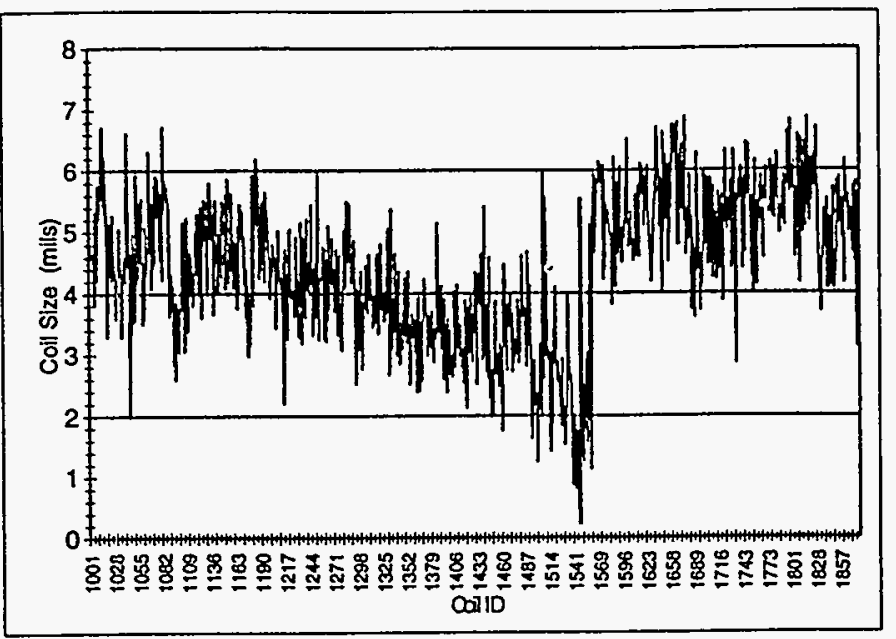

Fig. 7. RHIC $8 \mathrm{~cm}$ Quadrupole Mean Coil Azimuthal Size.

Each data point represents the overall average of many measurements taken along the coils, on both sides, at a constant pressure of $10 \mathrm{kpsi}(70 \mathrm{MPa})$. The nominal size values shown represent the difference from a machined standard gauge, which was fabricated to the magnetic design configuration, in thousandths of an inch, or mils. From a manufacturing standpoint, it was preferred to use constant cavity molding for both programs. Periodic adjustments to cavity size could be obtained by a variation in shim size thickness between the mating formblock and press tooling. An adjustment in shim size was performed at Dipole coil no. 1091. Although the data warranted a shim size change at coil number 1176 , it was avoided because of a planned wedge size change at coil no. 1211. It was anticipated that this wedge change would be sufficient to recover coil azimuthal size. As the data shows, the results were as expected and the proper coil size was regained.. For quadrupole coils, no change was made until coil no. 1563 to correct for an undersized coil condition. It should be noted that although the original goal of \pm 2 mils $( \pm 50 \mu \mathrm{m}$ ) of coil size variation has not always been met, the increase in allowed variation to \pm 3 mils in production has been tested to be completely acceptable in terms of mechanical magnet performance (magnetic performance is guaranteed by matching coils in any given cold mass to within 1 mil of each other in size) and allows for the simplicity of constant cavity molding.

Figs. 8 through 11 show straight section and end lengths of dipole and quadrupole coils. One impressive note is that the dipole straight section length largely meets requirements of \pm 0.040 inches $( \pm 1 \mathrm{~mm})$ despite the fact that the coil shrinks when removed from the centerpost tooling (due to metallurgical changes to the cable when heated) by more than .400 inches ( 1 $\mathrm{cm})$. The plots for quadrupole coil end length show a downward trend to an out of tolerance condition. This was traced to an improper method of fabricating coil end parts by a subcontractor. Afterwards, parts were $100 \%$ inspected and sorted at Northrop-Grumman and the condition disappeared. Lately, however, the condition has re-occurred despite inspection efforts. It is felt that the condition is due to all of the 


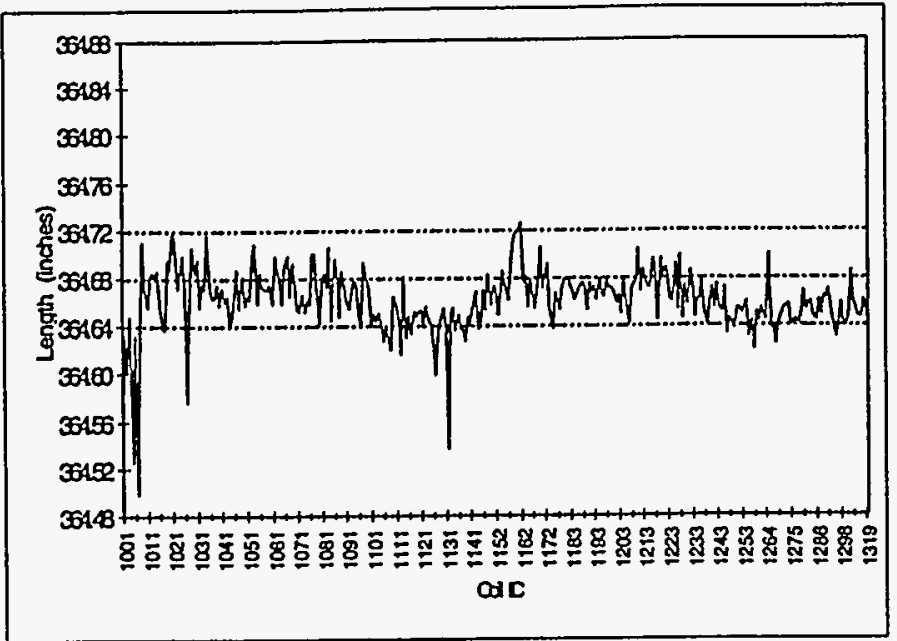

Fig. 8. RHIC $8 \mathrm{~cm}$ Dipole Coil Straight Section Length.

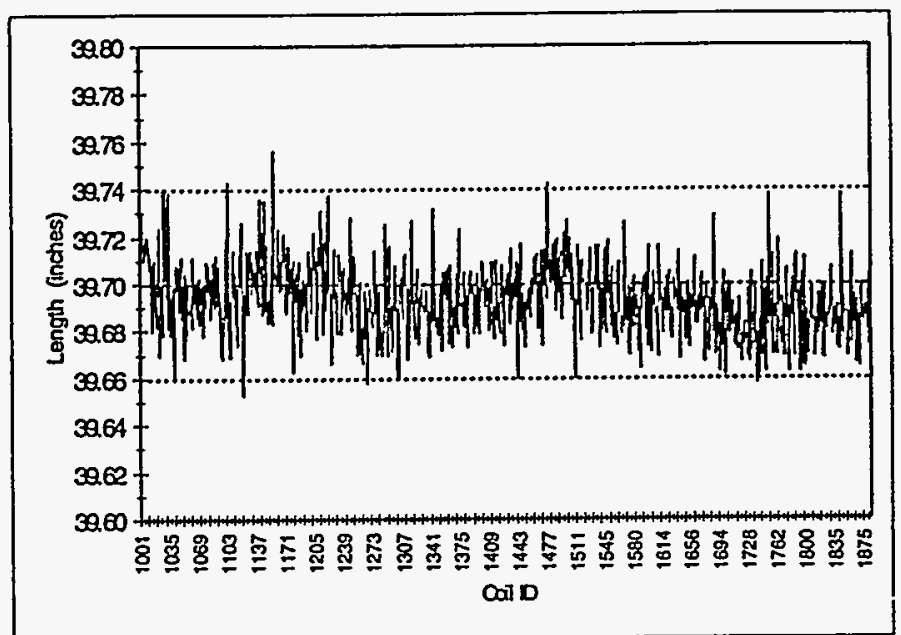

Fig. 9. RHIC $8 \mathrm{~cm}$ Quadrupole Coil Straight Section Length.

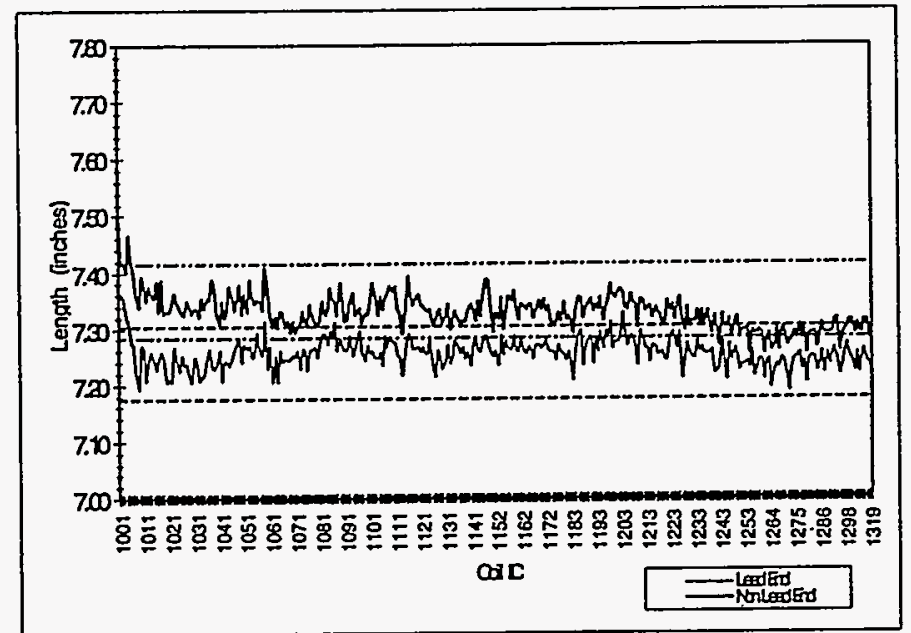

Fig. 10. RHIC $8 \mathrm{~cm}$ Dipole Coil Lead End and Non-Lead End Length.

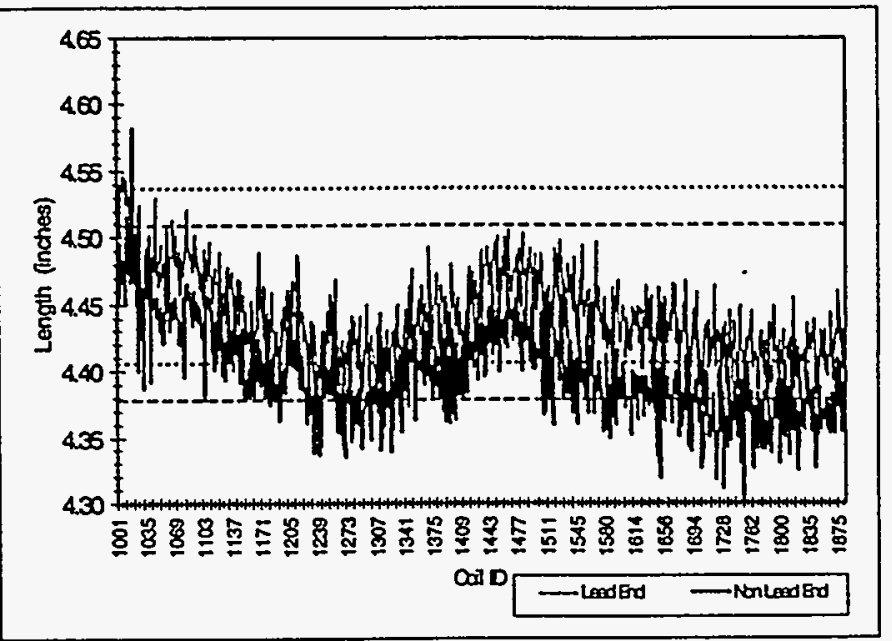

Fig. 11. RHIC $8 \mathrm{~cm}$ Quadrupole Coil Lead End and Non-Lead End Length.

coil end parts existing at the lowest allowable limit for size. Although this has been seen to affect the integral transfer function, the rms variation of the field integral is within tolerance and therefore no corrective action has been requested. For dipole coils, a similar condition has surfaced lately on nonlead coil ends, but as with quadrupole coils, no corrective action has been requested, although both situations continue to be monitored.

Figs. 12 and 13 show dipole and quadrupole collaring loads, respectively. Here, two separate values are monitored. The first is the load at which the yoke midplane closure has occurred. This is an empirical check of the final coil prestress, taking into account coil sizes, and insulator and yoke dimensional variations. It can be seen that problems developed at magnet sequence number 89 where a low value was first measured. This was correlated to magnetic measurements which indicated a shift in the magnetic transfer function

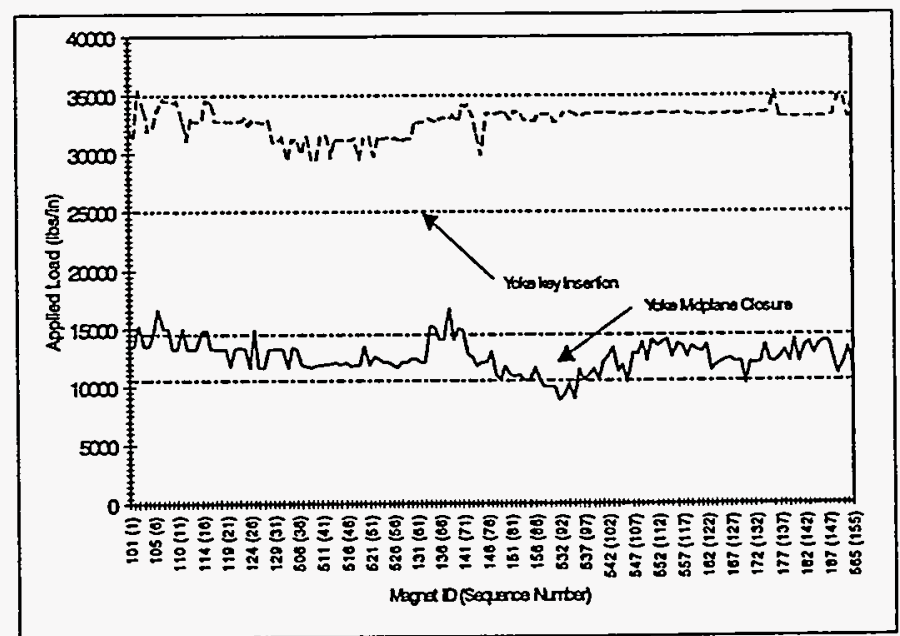

Fig. 12. RHIC $8 \mathrm{~cm}$ Dipole Collaring Data. 


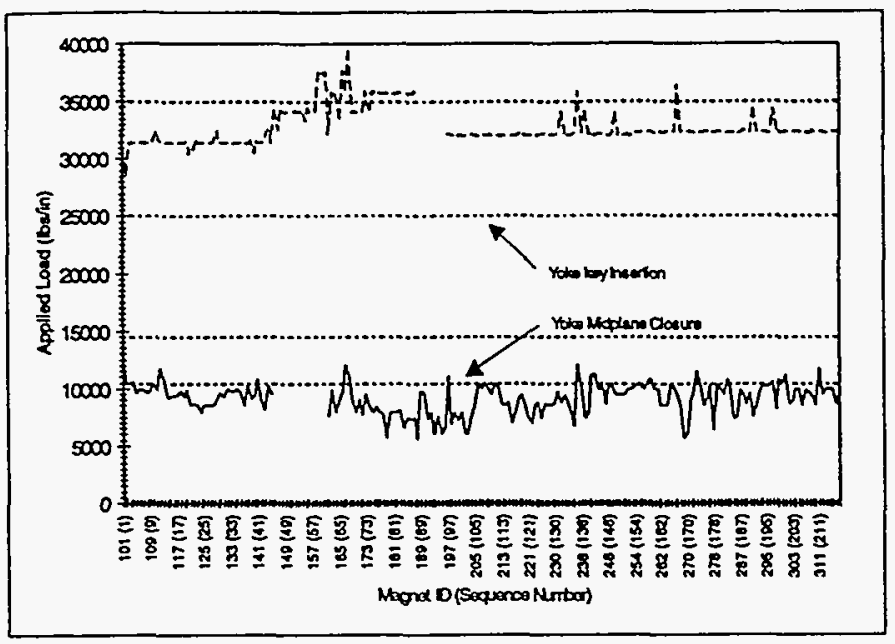

Fig. 13. RHIC $8 \mathrm{~cm}$ Quadrupole Collaring Data.

commensurate with radially undersized RX630 insulators [2]. This was confirmed by inspection and later traced to an improper repair made by the subcontractor to the insulator mold. Meanwhile, insulators were $100 \%$ inspected and sorted starting with magnet number 108 , until parts made after a proper mold repair could be introduced into magnets, starting with magnet number 137 . No problems have been encountered since, and source inspections and audits by NGC have been increased significantly at that subcontractor as a result.

The second value monitored is the load at final key insertion. This is greater than yoke midplane closure load since the yoke features a tapered midplane, and must be bent about itself much as a bolt is preloaded, to keep the midplane closed at the coil radius (for magnetic reasons) despite the force of the coils acting to try to open the midplane. No problems have been encountered with this parameter, despite some initial concerns of high loads required due to unexpectedly strong yoke steel.

Quadrupole collaring, by contrast, has been much more difficult. Initially, low yield strength yoke steel was used (by chance) in the laminations, and assembly was uneventful. Later, higher strength steel made assembly difficult despite going to higher load starting at cold mass number 46 . Part of the problem was due to the design of the press, which uses large cylinders spaced far apart. The lead end of the yoke, inherently weaker structurally due to cutouts for the coil leads, became overcompressed at higher load values. To compensate, NGC attempted to use an array of shims between press platens and on the cold mass directly, to restrict overcompression and gain mechanical advantage. Unfortunately, these shims both obscured the midplane closure pressure (observed to be low since the start of the program) and used a needed amount of press capacity. Various corrective actions, such as adding individual valves to each hydraulic cylinder, were recommended but not implemented due to schedule pressures. At cold mass number 62, all shims were removed and a different lot of yoke laminations with lower strength was used.
The higher strength steel has been segregated and is not presently intended to be used. Yoke midplane closure pressures continue to be monitored closely as the values remain low; however no corrective action has been requested since cold masses with similar values have tested to work extremely well.

Fig. 14 shows dipole cold mass sagitta values. The reported values for sagitta are derived from the inspection of the cold mass at eight locations, where access holes in the shell allow for inspection directly at the yoke survey notches. Although not shown in the plot, (and not planned for originally) approximately $95 \%$ of the sagitta is introduced during the shell welding operation. The remaining sagitta is added by reforming the cold mass in the inspection fixture.

Fig. 15 shows the cold mass twist values. Here, twist is the rms variation of the field angle, as derived from five measurements taken at the yoke survey notches through access holes in the shells. These measurements agree in general, although not perfectly, with the equivalent magnetic measurements for twist. Here, after some early problems., NGC was asked to do a complete analysis to determine the source of the occurrences of large twist. It was expected that a good correlation would be found between shell edge straightness and twist; however that was not the case. In fact, no strong conclusions were formed from the analysis. However, it seems that due to the increased awareness of the production staff, cold mass twist was improved significantly. Only when, for efficiency, the sagitta supports were increased to yield $100 \%$ sagitta after shell welding at magnet no. 83 has a systematic increase in twist occurred. This practice has been stopped and twist was reduced accordingly starting at magnet no. 91 .

\section{SUMMARY}

The industrial production of dipole magnets and quadrupole cold masses at NGC has been highly successful from the very beginning; magnets and cold masses meet or exceed requirements. The success can be attributed to the use

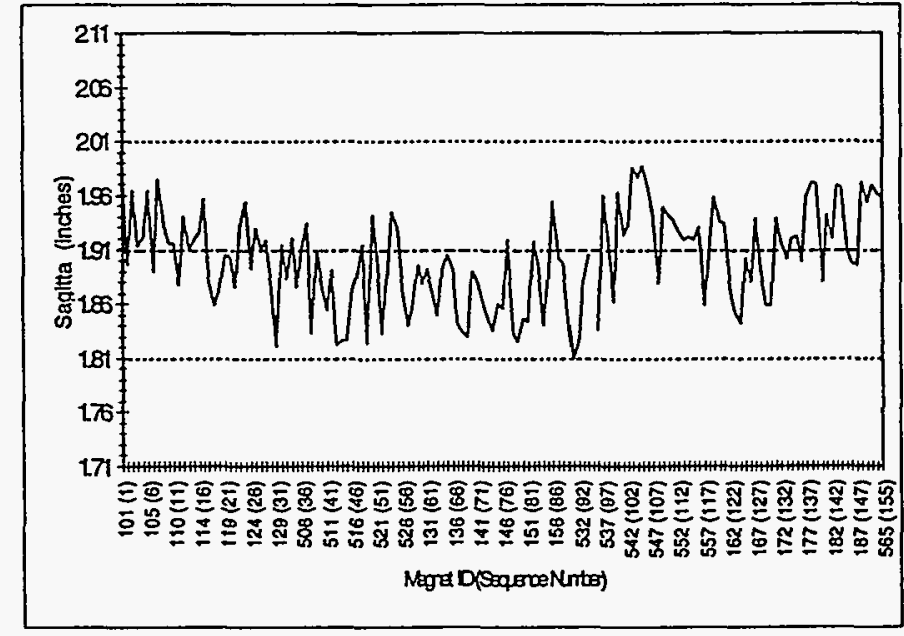

Fig. 14. RHIC $8 \mathrm{~cm}$ Dipole Derived Cold Mass Sagina. 


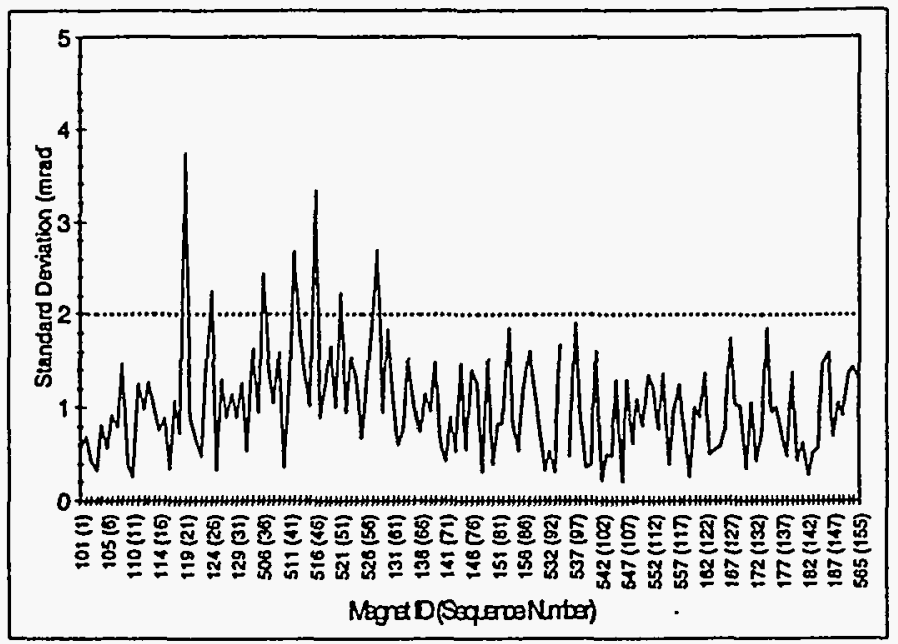

Fig. 15. RHIC $8 \mathrm{~cm}$ Dipole Twist Standard Deviation.

of proven magnet designs and construction methods established in advance by BNL, the proper utilization of all available resources both in manpower and equipment during Technology Transfer, and the construction of sensible tooling by NGC. Most importantly, the success can be attributed to the close working relationship between BNL and NGC on several levels, and the willingness to work together to identify and resolve problems as they surface.

\section{REFERENCES}

[1] P. Wanderer et al., "Technology Transfer for Industrial Production of Superconducting Magnets for the RHIC Project at BNL," Proc. Thirteenth International Conf. Jon the Application of Accelerators in Research and Industry, Denton, TX USA, Nov. 7-10, 1994 (to be published).

[2] P. Wanderer et al., "Construction and Testing of Arc Dipoles and Quadrupoles for the Relativistic Heavy Ion Collider (RHIC) at BNL". Proc. of the 1995 International Particle Accelerator Conference, Dallas, Texas, May 1-5, 1995.

[3] Kapton and Teflon are a registered trademark of the DuPont Corporation.

[4] M. Anerella et al., "Improved Cable Insulation for Superconducting Magnets," Proc. of the 1993 Particle Accelerator Conference (Washington, D.C.), pp. 2790-2792.

\section{ACKNOWLEDGMENTS}

The hard work, technical excellence, and tremendous dedication of the Northrop Grumman staff is thoroughly appreciated. Also, the help and guidance of the BNL RHIC Project technicians, design, and engineering staff during the Technology Transfer program is acknowledged, for laying the proper groundwork for a highly successful program. 